Instituto Internacional de Investigación y Desarrollo Tecnológico Educativo INDTEC, C.A.

DOI: https://doi.org/10.29394/scientific.issn.2542-2987.2017.0.0.11.194-214

OAI-PMH: http://www.indteca.com/ojs/index.php/Revista Scientific/oai

\title{
Herramientas de Comunicación Virtual para la Práctica Docente
}

Autor: Jerry Jesús Peña Morales

Universidad Politécnica Territorial de Trujillo "Mario Briceño Iragorry", UPTT "MBI"

jerryipm@gmail.com

Trujillo, Venezuela

\section{Resumen}

El objetivo del presente estudio es caracterizar las herramientas de comunicación virtual para la práctica docente en la Universidad Politécnica Territorial del Estado Trujillo, ubicada en parroquia Juan Ignacio Montilla, municipio Valera, estado Trujillo. Metodológicamente el estudio se enmarcó en una investigación documental con diseño de campo y tipo descriptivo. El aporte significativo es actualizar al docente o facilitador en las herramientas y en la importancia que estas revisten en los nuevos escenarios de formación virtual. Finalmente, este artículo considera la capacitación didáctica de los docentes o facilitadores hacia dichas herramientas.

Palabras clave: herramientas de comunicación virtual; formación online; práctica docente. 


\title{
Virtual Communication Tools for Teaching Practice
}

\begin{abstract}
The objective of the present study is to characterize the virtual communication tools for teaching practice at the Universidad Politécnica Territorial del Estado Trujillo, located in Juan Ignacio Montilla parish, Valera municipality, Trujillo State. Methodologically the study was framed in a documentary research with field design and descriptive type. The significant contribution is to update the teacher or facilitator in the tools and the importance they are in the new virtual training scenarios. Finally, this article considers the didactic training of teachers or facilitators towards these tools.
\end{abstract}

Keywords: virtual communication tools; online training; teaching practice. 


\section{Introducción}

En este momento, las Herramientas de Comunicación Virtual incursionan actualmente en todas las áreas de trabajo y en este mundo que cambia a pasos agigantados, también en el ámbito educativo hay un proceso de cambio y nos damos cuenta que necesitamos evolucionar ya que no solo el pizarrón, el proyector nos ayudan a facilitar la información, así que no podemos quedarnos atrás, la información la conseguimos mucho más rápido por la red a través de internet, las redes sociales, es por esto que se pretende dar a conocer cuáles son las herramientas de comunicación virtual para hacer el proceso educativo más dinámico, actualizar a los profesores y facilitadores en sus prácticas docentes.

En este momento, los programas educativos necesitan ser valorados en términos de eficacia y flexibilidad de aplicación en cuanto a tiempo, personal y recursos de que se disponga, lo cual exige al conocimiento de todo aquello que tiene que ver con la sociedad de la información, las nuevas tecnologías, la diversidad y diferenciación profesional, interacción de recursos. Así, los docentes, utilizando las nuevas tecnologías, pueden liberarse para realizar trabajos de orientación.

Los nuevos patrones didácticos en los que se tienen en cuenta las nuevas tecnologías y los medios de comunicación para optimar el aprendizaje suponen nuevas funciones de los docentes. Por esto, las nuevas realidades relacionadas a la transición hacia el tercer milenio en el deber ser que debe desempeñar la universidad, se vislumbran métodos de cambios acelerados e indecisión en todos los órdenes del acontecer humano.

En base a lo expuesto anteriormente, el objetivo de la indagación es caracterizar las herramientas de comunicación virtual para la práctica docente en la Universidad Politécnica Territorial del Estado Trujillo, ubicada en parroquia Juan Ignacio Montilla, municipio Valera, estado Trujillo. Por esto, se desarrolla la presente investigación en función de las herramientas de 
comunicación virtual para la práctica docente colocando de manifiesto la calidad, alcance que la revolución de la información y la comunicación tiene hoy mediante un futuro previsible para el desarrollo de la nueva sociedad, en la cual se espera que la universidad juegue un rol protagónico, de liderazgo en esta época de grandes transformaciones sociales de la humanidad.

\section{EI Problema}

El cómo funciona el ámbito educativo hoy, se vuelve apremiante en la práctica docente diaria y en contextos complejos, la necesidad de aprenderenseñar-aprender como un proceso recursivo. A la vez, requiere como profesional conocer cada una de ellas de manera individual logrando así efectividad en el trabajo realizado, por lo cual requiere de una preparación y conocimiento académico para lograr tal intento cuando se incrusta en el campo laboral educativo, siendo una de esas áreas la tecnología.

Desde esta perspectiva, la filosofía de la postmodernidad se supone como el primer fruto ocasionado por la sociedad tecnológica. Como lo expresa Lyotard, (1984) "al cambiarse las condiciones del saber cambia también no sólo el sentido del saber sino el saber mismo" (pág. 110). Ahora bien, esto en definitiva significaría aseverar que se ha transformado la sociedad, dado que en ella es que se produce el saber, entonces es de inferir que si cambia la sociedad no hay duda que cambian las condiciones desde las que emerge el saber. La situación descrita, ha dado motivo para el surgimiento de las nuevas tecnologías, las denominadas Tecnologías de la Información (TIC), las que han posibilitado tales transformaciones que han modificado la sociedad, y en consecuencia, las condiciones en las que se genera el saber.

Amparados en esta visión, el uso de las herramientas de comunicación virtual para la práctica docente en la educación, tanto en la automatización de sistemas de información y administrativos como para la promoción del aprendizaje, ha tenido en las tres últimas décadas buena acogida. Sin 
embargo, como problema, se percibe poca comprensión en los diversos niveles de las comunidades educativas sobre las dinámicas de inserción de lo digital en el aula presencial.

Por esto, en el presente trabajo lo que se pretende, es mostrar estas herramientas de comunicación virtual para que el profesor o facilitador pueda utilizarlas con sus estudiantes y trabajar a través de la red. Esto motivado a que, sin duda internet se ha convertido en una de las tecnologías de suma importancia en la sociedad del conocimiento. Y esto influye en la educación de cualquier nivel, tanto en la modalidad de formación online (en línea) como semipresencial. Por esto, se plantea la interrogante ¿Cuáles serían las herramientas de comunicación virtual a incluir en la práctica docente?

\subsection{Objetivos de la Investigación}

\subsubsection{Objetivo General}

Caracterizar las herramientas de comunicación virtual para la práctica docente en la Universidad Politécnica Territorial del Estado Trujillo, ubicada en parroquia Juan Ignacio Montilla, municipio Valera, estado Trujillo.

\subsubsection{Objetivos Específicos}

Mostrar las herramientas de comunicación virtual que el profesor o facilitador puede incorporar en su práctica docente.

Desarrollar ejemplos prácticos sobre cómo incorporar las herramientas de comunicación virtual en su práctica docente.

\section{Referentes Teóricos}

\subsection{Herramientas de Comunicación Virtual}

En la actualidad, el docente de hoy puede encontrarse con un volumen de herramientas de comunicación tan amplio que no es fácil, en ocasiones, poder estar al día en lo que a su incorporación didáctica se refiere. En este 
sentido, se pueden hallar de diferente tipología, como las referentes a la instrucción, a la tutoría virtual, o a otros campos educativos, ampliándose en los últimos años debido a los cambios tecnológicos, así como al movimiento del software libre, y a la aparición de nuevas formas de entender la formación soportada en la red. La comunicación podemos establecerla de manera sincrónica que permite la interacción en distinto espacio, asimismo asincrónica porque facilita la interacción en distinto espacio y en distinto tiempo.

Aunado a esto, hay que hacer referencia a Cabero, (2006), donde establece "las bases pedagógicas de la formación online y las variables para obtener resultados significativos son" (pág. 67):

- Competencias tecnológicas.

- E-actividades.

- Soporte institucional y aspectos organizativos.

- Papel del profesor/facilitador.

- Papel del estudiante.

- Centrado en el estudiante, activo, colaborativo y participativo.

- Contenidos.

- Sentido de comunidad, sociabilidad, interactividad social.

- Metodología, diseño y estrategias didácticas.

No se va profundizar en cada una de ellas, pero sí centrar específicamente en las que están vinculadas con el objeto de análisis del presente artículo. Uno de los requisitos indispensables para que una acción formativa soportada en la red sea de calidad, es el buen uso y el dominio tecnológico y didáctico que el docente o facilitador posea con respecto a la utilización de las diferentes herramientas de comunicación virtual disponible. A continuación, se visualiza el cuadro $\mathrm{N}^{\circ} 1$, mostrando un ejemplo de las diferentes herramientas de comunicación virtual que más empleadas en red. 
Cuadro $\mathbf{N}^{\circ}$ 1. Herramientas de Comunicación Virtual.

\begin{tabular}{|l|l|}
\hline \multicolumn{1}{|c|}{$\begin{array}{c}\text { Herramientas de Comunicación } \\
\text { Sincrónicas }\end{array}$} & \multicolumn{1}{c|}{$\begin{array}{c}\text { Herramientas de comunicación } \\
\text { Asincrónicas }\end{array}$} \\
\hline Chat (IRC). & $\begin{array}{l}\text { Foros o grupos de noticias. } \\
\text { TV-web (video streaming). } \\
\text { Videoconferencia } \\
\text { Audio conferencia. }\end{array}$ \\
MUD (Multi-user dimensions). & Cobates telemáticos. \\
Pizarras electrónicas compartidas. & Correos de voz (voice- mail). \\
Navegación compartida & Correos de vídeo (video- mail). \\
& Herramientas para el trabajo \\
& colaborativo. \\
& Plataformas de Aprendizaje \\
& Wiki, Blog \\
& Poscast \\
\hline
\end{tabular}

Fuente: El autor, (2016)

Por ende, las herramientas de comunicación virtual nos facilitan realizar una comunicación textual, auditiva, visual y audiovisual. Así mismo, nos ayuda a realizar diferentes tipos de actividades, que pueden ir desde impartir formación, realizar tutorías, efectuar actividades de tipo colaborativo entre los estudiantes o participantes. A continuación, se muestran algunas de las herramientas de comunicación virtual que el profesor o facilitador puede incorporar en su práctica docente.

\subsection{El Correo Electrónico.}

Aunque ahora tiene un gran competidor como son las redes sociales, quizás sea el correo electrónico la herramienta de comunicación que más ha tenido auge en lo que a tecnología de la comunicación se refiere. Y ello, debido fundamentalmente, a las grandes posibilidades comunicativas y didácticas que nos ofrece, que puede ser, en primer lugar, la de facilitarnos una conversación más abierta, entre los profesores o facilitadores y estudiantes, lo que parecía impensable en épocas anteriores en situaciones de aprendizaje de carácter presencial. 
Algunos estudios demuestran que los estudiantes se sienten algunas veces más cómodos a formular preguntas y dudas a los profesores a través del correo electrónico que en una situación de presencialidad, de forma que los profesores o facilitadores son percibidos como más accesibles. Al respecto, según Valverde (2002) "hay una serie de recomendaciones para conseguir un uso más eficaz del correo electrónico" (pág. 62) donde se encuentran las siguientes:

- Leer el correo con regularidad y responder con celeridad posible.

- Identificar el asunto.

- Identificarse como emisor.

- Cuidar la expresión y la ortografía.

- Ser preciso en las solicitudes y en las contestaciones.

- Usar en la respuesta parte del texto recibido.

- Controlar el tamaño de los mensajes.

- Solicitar información sobre la recepción de información.

Aunque en ocasiones, cuando estamos empleando el correo electrónico en procesos formativos online debemos prestarle una especial atención, ya que ello nos va a permitir, por un lado, poder identificar el tema sobre el que los estudiantes hacen referencia, y por otro lado, poder organizarlos en carpetas, lo cual nos facilitará el poder encontrar cualquier mensaje si deseamos retomarlo con posterioridad para su consulta.

\subsection{La Mensajería Instantánea como Recurso Educativo}

El poder comunicarnos de manera simultánea y sincrónica ha supuesto un gran cambio para todos los que formamos parte de la red, más cuando nos referimos al ámbito educativo online, donde su incorporación supuso un avance en lo que a los procesos comunicativos se refiere. La utilización de la 
mensajería instantánea puede realizarse a través de diferentes programas, desde aquellos que exigen un costo hasta los que se distribuyen de forma gratuita en Internet.

Tal como nos indican Ortega y otros (2000), "la comunicación a través de la mensajería instantánea ofrece diferentes posibilidades educativas" (pág. 3839) que concretan en las siguientes:

- Favorece la innovación educativa,

- Estimula la actualización de los profesores,

- Es útil en la formación y el perfeccionamiento del profesorado,

- Es un instrumento para el trabajo en equipo, y propicia una actitud abierta al uso de las nuevas tecnologías.

La comunicación a través de un chat ha ido evolucionando de manera vertiginosa en los últimos años, en los comienzos de su aparición era frecuente la utilización del IRC, o del "MSN Messenger", aunque en la actualidad aparecen nuevas herramientas de comunicación vinculadas con el movimiento del Mobile learning. Con ello, nos venimos a referir al uso que los estudiantes realizan de herramientas de comunicación para el móvil como el "WhatsApp", disponible para iPhone, BlackBerry, Samsung y teléfonos inteligentes y también ha propiciado el menor número de envíos de SMS.

Sin embargo, hay algunas limitaciones, y que como apunta Pérez, (2006), "a veces provoca que los sujetos que participan en la conversación, en este caso profesores y estudiantes, puedan perderse en lo que respecta al diálogo establecido" (pág. 195). Seguidamente, se puede visualizar un ejemplo práctico, de un modelo establecido de planificación que puede ayudar al profesor o facilitador a orientarse cómo puede incorporar las herramientas de comunicación virtual en su práctica docente, al momento de Planificar un chat como se ve en el Cuadro $\mathrm{N}^{\circ} 2$, a continuación: 
Cuadro $\mathbf{N}^{\circ}$ 2. Planificación del Chat sugerido.

\section{Preliminares:}

a) El Chat en cuestión está pautado para el día miércoles 14 de julio a las 10:00 A.M (hora de Venezuela), 09:00 A.M (hora de Boston) y 3:00 P.M (hora de España) y tendrá una duración de sesenta minutos; es decir, una hora.

b) La ruta de acceso a la web desde dónde se realizará el Chat es la siguiente:

Primero se busca la dirección http://www.iutet.edu.ve/

Segundo, se oprime el botón "Aula Virtual" ubicado en parte inferior izquierda de la pantalla;

Tercero, se oprime el botón "Login";

Cuarto, se introduce el Username o nombre de usuario y el Password o contraseña respectivamente;

Quinto, se selecciona la opción "Informática II" que está ubicada en la sección correspondiente a "Mis Cursos" en el extremo derecho de la pantalla;

Sexto, seleccionamos la opción "Comunicaciones" entre las actividades básicas que ofrece el Aula en la columna de la izquierda;

Séptimo, se hace clic sobre "Virtual Classroom";

Octavo, se selecciona la opción "Enter Virtual Classroom", esperamos que cargue el programa y habremos llegado al lugar desde donde se realizará el chat.

c) Los actores del Chat se han dividido en tres grupos atendiendo a la naturaleza de su participación, así tenemos: Los invitados (los expertos en el tema Profesores Reina Caldera y Carlos La Cruz); El Moderador (Prof. Jerry Peña) y Los Estudiantes (que son los integrantes del curso participante y que a su vez, se han organizado en tres grupos para formular los planteamientos a considerar). 
d) El Moderador les hará llegar con antelación a cada actor involucrado en el Chat, su "Username" y su "Password" de acceso al Aula Virtual.

e) Una vez distribuidos los "Username" y los "Password" entre los actores del Chat, el Moderador propiciará conexiones previas con los involucrados a fin de verificar el acceso y la fluidez del mensaje en el medio.

f) El día del Chat (14/07/14), El Moderador se conectará 15 quince minutos

g) El término clave para declarar finalizada una intervención durante la ejecución del Chat será el adverbio circunstancial de lugar: "ADELANTE".

\section{Guión del Chat:}

1. Una vez chequeada la asistencia de los actores involucrados, el Moderador declarará "Abierto El Chat".

2. Acto seguido, El Moderador da la Bienvenida Oficial a los Invitados y cede el turno al Dr. Carlos La Cruz para que responda al saludo.

3. La Dra. Reina Caldera responde el saludo.

4. El Moderador le cede el tumo a la Dra. Reina Caldera

5. La Dra. Reina Caldera responde al saludo.

6. El Moderador confirma que los planteamientos están dirigidos por igual a ambos invitados, los cuales se irán alternando en el orden de respuesta, correspondiendo el inicio al Dr. Carlos La Cruz. Seguidamente, el Moderador cede el turno al estudiante representante del primer grupo para que formule su planteamiento.

7. El estudiante escribe su planteamiento.

8. El Dr. Carlos La Cruz responde.

9. La Dra. Reina Caldera responde.

10. El Moderador cierra el planteamiento y cede el turno al estudiante representante del segundo grupo para que formule su planteamiento. 
11. El estudiante escribe su planteamiento.

12. La Dra. Reina Caldera responde.

13. El Dr. Carlos La Cruz responde.

14. El Moderador cierra el planteamiento y cede el turno al estudiante representante del tercer grupo para que formule su planteamiento.

15. El estudiante escribe su planteamiento.

16. El Dr. Carlos La Cruz responde.

17. La Dra. Reina Caldera responde.

18. El Moderador cierra el planteamiento y chequea el tiempo disponible. De quedar más de quince minutos disponibles dentro de la hora pautada para el Chat, El Moderador cederá

el turno al estudiante encargado de repreguntar sobre las respuestas emitidas por los Expertos Invitados.

19. El estudiante escribe su repregunta e indica a quién de los dos Invitados va dirigida.

20. El Invitado afectado responde.

21. El estudiante escribe su repregunta al otro Invitado.

22. El Invitado afectado responde.

23. El Moderador agradece la participación de los actores y en especial la de los Invitados y les cede el turno a estos últimos para que se despidan comenzando por el Dr. Carlos La Cruz.

24. El Dr. La Cruz se despide.

25. La Dra. Caldera se despide.

26. El Moderador declara cerrado oficialmente el chat.

Fuente: El autor, (2016).

El esquema anterior, se hace más necesario si involucramos a los estudiantes a utilizar esta herramienta de comunicación virtual para que se 
sientan de una forma amena y agradable. Debemos de ser conscientes, que uno de los problemas que presenta esta herramienta es la ausencia de información extralingüística. $Y$ aunque, existen una serie de alternativas, que van desde el escribir las emociones hasta la utilización de emoticonos (dibujos que expresan emociones) que pueden resolver este problema.

La realidad es que tenemos que contemplar que perdemos esta dimensión y también la de las referencias de comunicación no verbal. Algunas de las posibilidades didácticas que esta herramienta nos ofrece, según Sánchez y Soto (2006) "dependerá en gran medida del buen uso en el que el docente y los estudiantes sean capaces de realizar con ella" (pág. 81). A continuación, apuntamos algunos de los elementos que intervienen en un enfoque didáctico del chat.

- Variable contextual estudiante: uso habitual del chat, actitud y predisposición, habilidad con el teclado, lugar de acceso al chat, participación e interacción.

- Planificación de la sesión: objetivos y contenido de la sesión, normas y reglas de uso, existencia de un moderador, estructura de la comunicación, temporalización, número de participantes, evaluación.

- Texto de las intervenciones: preparación de intervenciones, fluidez, relevancia y coherencia con el tema de la conversación, uso adecuado del lenguaje, textos claros y sintéticos, creatividad y expresividad.

- La herramienta: acceso, capacidad y conexión y otras herramientas.

Es importante apuntar, según Llorente, (2009) "diferentes aspectos que deben ser contemplados por el profesor o facilitador en el proceso de la práctica docente utilizando la herramienta del chat" (pág. 72), a saber:

1. Ser puntual en el inicio de la sesión.

2. Recordar las recomendaciones de la participación. 
3. Es aconsejable emplear frases cortas cuando se redacta en una sesión de chat.

4. Cuidar la redacción y la ortografía, de manera que sean comprensible para todos los participantes en la sesión.

5. Las mayúsculas, en la comunicación por chat, indican estar gritando, por lo que es recomendable no emplearlas sólo cuando las reglas ortográficas tradicionales lo apuntan.

6. Mantener el hilo conductor, e intentar que el tema no se desvíe hacia otros durante la conversación.

7. Evitar las cuestiones personales, y conseguir que todos se beneficien de los temas que se están tratando.

8. Valorar la capacidad de síntesis en los argumentos.

9. No intervenir mientras otra persona tenga la palabra, lo que facilitará la lectura de los demás participantes, tomar nota si luego se quiere comentar.

10. Finalizar cada sesión con un resumen de lo tratado y conclusión.

11. Finalizar a la hora prevista.

Por último, haciendo referencia a las diferentes posibilidades y limitaciones que esta herramienta nos proporciona en su incorporación al ámbito educativo en procesos de formación universitarios a través de la red, y que exponemos a continuación.

Ventajas: a). De fácil acceso; b). Útil para crear un espacio de reunión a distancia entre personas con los mismos intereses; c). Flexibilidad para interactuar fuera del horario presencial y d). Comunicación en tiempo real y desde espacios geográficos diferentes.

Limitaciones: a). Rapidez de la comunicación; b). Necesidad de competencias tecnológicas para su incorporación y c). Excesiva planificación de la comunicación. En otras palabras, cuando empleamos la herramienta chat con fines educativos una variable influyente es el número de personas que 
participan en la conversación, ya que si el número es elevado la calidad de la comunicación y la conversación disminuye que si lo realizamos con grupos más pequeños. Aun así, esta herramienta de comunicación continúa siendo para el docente o facilitador un medio significativo por tres grandes motivos: distribución e intercambio de información, posibilidad de poner en práctica el aprendizaje colaborativo, y socializar a estudiantes en online.

\subsection{Los Foros de Discusión y los Grupos de Noticias.}

La diferencia entre una herramienta y otra, es que los foros de discusión se refieren a listas de correo electrónico tematizadas a las cuales se suscribe el usuario o los crea el docente en una plataforma específica, y los grupos de noticias son tablones donde los profesores pueden incorporar mensajes $u$ opiniones. Lo que las hace similares es que las dos pueden ser empleadas para intercambiar opiniones entre profesores o facilitadores y estudiantes, establecer contacto, diseñar y trabajar en proyectos comunes, solicitar asesorías u orientación, analizar diferentes perspectivas.

En lo que respecta a los foros de discusión, a juicio de Mc Elhem (1996) "éstos pueden ser seleccionados por el usuario, o específico creado por el docente o facilitador" (pág. 21), por esto, según el autor mencionado podemos establecer una primera clasificación de acuerdo a diferentes tipos de criterios, que serían básicamente.

- Temática: de interés general, en temas concretos y especializadas.

- Accesibilidad: públicas y privadas.

- Moderación: moderadas y no moderadas.

- Distribución: unidireccionales y multidireccionales.

- Orientación: ubicada a los productos y a los procesos.

Por el contrario, si hablamos de los grupos de noticias Valverde (ob.cit) 
64) señala que éstos "se configuran como una comunidad virtual que se basa en el principio de dar tanto como se obtiene, de tal modo que un usuario de news debería de mantener una actitud activa" (pág. 64), es decir, se debe aportar cuando sea necesario su experiencia o conocimiento sobre el tema a través del envío de mensajes.

Pero, basándonos en qué papel juega el rol del docente o facilitador en cada uno de ellos, podemos apuntar que éste es de vital importancia, y que su capacitación tanto tecnológica como didáctica debe ser alta, habilidades que pueden concretarse en: mantener un estilo de comunicación no autoritario, tener claro objetivos de participación, ser objetivo y considerar el tono de la intervención, promover conversaciones privadas, alabar y reforzar públicamente conductas positivas, cuidar el humor y sarcasmo, no ignorar las conductas negativas, llamar la atención de forma privada.

De acuerdo a lo planteado se alude la postura de Berge, (1995) donde expone "tanto en una como en otras el papel del profesor o facilitador es de máxima importancia, y para cumplirla deberá de tener una serie de habilidades y capacidades" (pág. 30). A continuación, las siguientes:

- Tener claro los objetivos de la participación.

- Mantener un estilo de comunicación no autoritario.

- Ser objetivo y considerar el tono de la intervención.

- Promover conversaciones privadas: diseñar situaciones con intereses similares.

- Presentar opiniones conflictivas.

- Cuidar el uso del humor y del sarcasmo, ya que no todo el mundo puede compartir los mismos valores.

- Alabar y reforzar públicamente las conductas positivas.

- No ignorar conductas negativas, llamar la atención de forma privada. 
Además, reiteramos que en los procesos de la práctica docente el facilitador o docente debe desarrollar una serie de competencias a la hora de incorporar herramientas de comunicación virtual, con en este caso son el foro de discusión y los grupos de noticias, y que se refieren a la planificación (determinar el tiempo que durará la actividad, seleccionar material de trabajo, e informar a los estudiantes), tiempo de trabajo de los alumnos, desarrollo de la sesión, conclusiones, evaluaciones. Presentamos, a continuación, según Anderson (2005) son diferentes fases "a la hora del análisis de la interacción y la construcción del conocimiento a través de la incorporación de los foros de discusión" (pág. 65):

Fase 1: Compartir y comparar información: manifestar una observación, de acuerdo a uno o más participantes, corroborar ejemplos, preguntas, respuestas, clarificar detalles, definición, descripción de un problema.

Fase 2: Descubrir, explorar disonancias o debilidad de ideas, conceptos o enunciados: se caracteriza por la identificación de desacuerdos, preguntas y respuestas para clarificar el origen del desacuerdo, citas bibliográficas, experiencia, propuesta para apoyar argumentos.

Fase 3: Negociar significado/co-construcción cooperativa de conocimiento: se caracteriza por la clarificación de significados, negociación e importancia de los argumentos, identificación de áreas de acuerdos contra desacuerdos, propuesta nuevas negociaciones de creencias que encierran compromisos y co-construcción de conocimiento y/o propuesta de integración de metáforas y analogías.

Fase 4: Prueba y modificación de síntesis propuesta o co-construcción: someter a prueba y modificar la síntesis co-construida, comprobar la síntesis propuesta para un esquema cognitivo existente, comprobar contra experiencias personales, evidenciar contra datos formales y demostrar la síntesis propuesta contra testimonios contradictorios dentro de la literatura. 
Fase 5: Enunciar acuerdos y aplicar nuevos significados construidos: acuerdos y aplicaciones que integren los diferentes convenios, que apliquen nuevos conocimientos y que se hagan reflexiones metacognitivas que ilustran el entendimiento y el cambio de las formas de pensamiento como resultado de la interacción.

\section{Metodología}

En toda investigación, según Malavé, (2003) se describe que la metodología "es el fundamento para el desarrollo del trabajo de exploración propiamente dicho" (pág. 57). Esto corresponde, a ser la guía para conseguir los datos necesarios a los que recurrió el autor y así insertarlos en la teoría para sustentar la temática tratada. Por esto, básicamente la investigación es documental en este orden, según Arias, (2006):

La investigación documental es un proceso basado en la búsqueda, recuperación, análisis, crítica e interpretación de datos secundarios, es decir, los obtenidos y registrados por otros investigadores en fuentes documentales: impresas, audiovisuales o electrónicas. Como en toda investigación, el propósito de este diseño es el aporte de nuevos conocimientos. (pág. 27).

De hecho, en la indagación documental, el investigador debe tener habilidades para proveerse de escritos preliminares significativos que le ofrecerán soporte para relacionarlos con los datos que va consiguiendo en su estudio, aunado a un buen análisis que haga de éstos y su paráfrasis le guie hacia la obtención de sus objetivos específicos y logre el objetivo general.

\section{Conclusiones}

Para finalizar, creemos conveniente que la incorporación de los foros de discusión en la práctica docente conlleve también un análisis del discurso o la comunicación que se produce en ellos, para poder determinar los usos que se 
realizan de los mismos, interacciones, sugerencias, etc. Es significativo comprobar la rapidez con la que surgen nuevas herramientas de comunicación virtual disponibles en la red, y la rapidez con la que estudiantes adquieren competencias suficientes para desenvolverse con soltura ante las mismas. Pero queremos hacer hincapié en una cuestión que nos parece de especial relevancia: la capacitación didáctica hacia dichas herramientas.

Es obvio que los estudiantes pueden emplearlas desde una perspectiva de ocio y diversión, pero resulta necesario por parte del profesor planificar y gestionar su incorporación desde un prisma educativo. Por otro lado, la siempre necesaria capacitación técnica y didáctico-curricular del docente o facilitador en el uso e incorporación de dichas herramientas. Por último, se hace necesaria la difusión de estudios e investigaciones relacionadas con los procesos de la práctica docente a través de la red, y de cómo los profesores incorporan las diferentes herramientas de comunicación y con qué fines educativos.

\section{Referencias}

Anderson, T. (2005). El E-learning en el Siglo XXI. Investigación y Práctica. Barcelona. Ediciones Octaedro.

Arias, F. (2006). El Proyecto de Investigación. Introducción a la Metodología Científica Quinta edición. Caracas: Editorial Episteme Berge, Z. (1995). "Facilitating computer conferencing: recommendations from the field". Educational Technology, 35, 1, 22-30.

Cabero, J. (2006). "Las Herramientas de Comunicación en el Aprendizaje

Mezclado". Píxel-Bit. Revista de Medios y Educación, 23, 27-41.

Lyotard, J. (1984). La condición posmoderna, Edición. Cátedra, Madrid.

Malavé, L (2003). EI Trabajo de Investigación. Quirón Ediciones. Caracas.

Ortega, F. y otros (2000). "EI IRC como Herramienta para la Formación

Flexible y a Distancia". Pixel Bit. Revista de medios, educación, 31. 
Pérez, A. (2006). Internet Aplicado a la Educación: Aspectos Técnicos y Comunicativos. Las plataformas, en Cabero, J. (coord). Nuevas tecnologías aplicadas a la educación. Madrid: McGraw-Hill, 189-203.

Sánchez Soto, J. (2006). El Chat en la Tele Enseñanza: Implicaciones Comunicativas y la Oportunidad de un Enfoque Didáctico. Actas del Congreso Internacional Edutec 2005. Formación del profesorado y nuevas tecnologías. Santo Domingo (República Dominicana).

Valverde, J. (2002). Herramientas de Comunicación Sincrónica y Asincrónica, en AGUADED, J. I. y CABERO, J. (dirs). Educar en red. Málaga: Aljib. 
Jerry Jesús Peña Morales

e-mail: jerryjpm@gmail.com

Nacido en Caracas, Venezuela. Graduado en Ingeniería

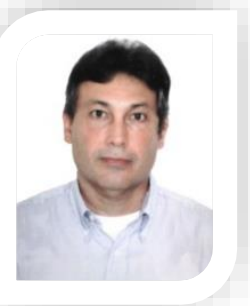
de Sistemas, cursado en Universidad de Los Andes. Actualmente curso estudios de Doctorado en Ciencias de la Educación en Universidad Nacional Experimental "Rafael María Baralt". Magister en Ingeniería de Control de Procesos en Universidad Experimental Politécnica.

Barquisimeto. Experiencia Laboral: Actualmente en Instituto Universitario de Tecnología del Estado Trujillo. Cargo: Docente Contratado (Tiempo Completo) Desde Abril 1998. Asesor de Sistemas de Información y programación. Además, puedo capacitar a cualquier personal en el área de ofimática (procesador de texto, hoja de cálculo, presentaciones) y en el área de informática en el sistema operativo Windows y Linux.

El contenido de este manuscrito se difunde bajo una Licencia de Creative Commons ReconocimientoNoComercial-Compartirlgual 4.0 Internacional 